\title{
Simulations of the IMF in Clusters
}

\author{
Ralph E. Pudritz \\ Origins Institute, McMaster University, ABB 241, \\ Hamilton, Ontario L8S 4M1, Canada \\ email: pudritz@mcmaster.ca
}

\begin{abstract}
We review computational approaches to understanding the origin of the Initial Mass Function (IMF) during the formation of star clusters. We examine the role of turbulence, gravity and accretion, equations of state, and magnetic fields in producing the distribution of core masses - the Core Mass Function (CMF). Observations show that the CMF is similar in form to the IMF. We focus on feedback processes such as stellar dynamics, radiation, and outflows can reduce the accreted mass to give rise to the IMF. Numerical work suggests that filamentary accretion may play a key role in the origin of the IMF.
\end{abstract}

Keywords. initial mass function, turbulence, accretion, filaments, magnetic fields, feedback

\section{Introduction}

The Initial Mass Function (IMF) plays a central role in astrophysics because it encapsulates the complex physics of star formation. Observations suggest that the IMF can be variously described as a piece-wise power-law (Kroupa 2002), a lognormal (Miller \& Scalo 1979), or a lognormal distribution with a high-mass power-law tail (Chabrier 2003). The high mass behaviour of the IMF Salpeter (1953) is a power-law, $d N \propto m^{-2.3} d m$ for stellar masses $m \geqslant 0.5 M_{\text {odot }}$. The peak mass for isolated stars in the galactic disk is $0.1 M_{\odot}$ and $0.2-0.3 M_{\odot}$ (Chabrier 2003) for the bulge. The form of the IMF is similar in many different galactic and extragalactic environments such as globular clusters, wherein one has a large range of metallicities and concentrations (Paresce \& De Marchi 2000). The current evidence therefore tends to support the notion that the IMF is universal.

What physical processes produce the IMF? Observations show that it emerges during the early stages of the formation of star clusters (Meyer et al. 2000; Zinnecker et al. 1993). Young stars are formed within gravitationally bound subunits of a cluster-forming environment known as "cores" whose mass distribution - the core mass function (CMF) - strongly resembles the IMF. Cores are closely associated with filaments, as recent observations using the Spitzer and Herschel observatories clearly show (André et al. 2010). Competing physical processes such as turbulence, gravity, cooling and thermodynamics, as well as magnetic fields play significant roles in building the CMF, as well as filaments, within molecular clouds. Feedback processes such as radiation from massive stars, jets and outflows, as well as stellar dynamics serve to truncate the accretion of material onto stars and their natal disks. These may lead to the emergence of the IMF from the CMF.

This review focuses on the critical role that computation is playing in exploring the origin of the IMF in clusters. We focus first on the processes leading to the CMF, and then discuss feedback processes that may convert the CMF into the IMF. Recent reviews of the computational aspects of the IMF may be found in Mac Low \& Klessen (2004), Bonnell et al. (2007), Larson (2007), and Klessen et al. (2009). The theory of the IMF is covered by Hennebelle (this volume), and McKee \& Ostriker (2007). 


\section{Structure formation and the IMF}

Structure formation in the diffuse ISM as well as the dense molecular medium is a shocking affair. Diffuse atomic hydrogen near the midplane of our Milky Way is observed to be organized as a plethora of filamentary structure, bubbles, supernova remnants, HII regions (Taylor et al. 2003). Filamentary structure also characterizes giant molecular clouds - as seen in the large scale extinction maps of the Orion and Monocerus clouds (Cambrésy 1999). On smaller scales, a 850 micron continuum map of a 10pc region in Orion shows that Bonner-Ebert like cores are associated with filaments (Johnstone \& Bally 2006). Herschel observations, such as those of the IRDC filament known as the "snake", show massive stars and a star cluster in formation (Henning et al. 2010).

How can cloud structure be characterized? One fruitful approach is to measure the probability distribution functions (PDFs) of the cloud column density of all of the gas in a given molecular cloud which is readily measured directly from the extinction data. This PDF for clouds without star formation (such as Lupus V and the Coal Sack clouds) turns out to be lognormal, whereas that of star forming clouds is a lognormal plus high mass, power-law tail (Kainulainen et al. 2009). This is interpreted as arising from the effects of gravity which drives collapse.

Filaments have various origins and arise in supersonic turbulent media due to the intersection of shocks waves, by gravitational break-up of self-gravitating sheets, or by thermal instabilities of various kinds. A physically plausible picture for the origin of the CMF is that filaments with sufficiently high values of their mass per unit length produce cores by gravitational instability. Gravitational fragmentation of filaments is well studied theoretically (Nagasawa 1987; Fiege \& Pudritz 2000) but has received renewed emphasis with the Herschel observations.

The similarity in the functional form of the CMF and the IMF has been observed in many clouds starting with the study of Motte et al. (1998) in $\rho$ Oph. The CMF in the Pipe Nebula, as another example, can be shifted into the IMF by converting a fraction $\epsilon \simeq 1 / 3$ of its mass to stars Alves et al. 2007 .

\section{From clouds to the CMF}

\subsection{Turbulence}

Supersonic turbulence rapidly compresses gas into a hierarchy of sheets and filaments wherein the denser gas undergoes gravitational collapse to form stars (Porter et al. 1994; Vazquez-Semadeni et al. 1995; Ostriker et al. 1999; Klessen \& Burkert 2001; Padoan et al. 2001; Bonnell et al. 2003; Tilley \& Pudritz 2004; Krumholz et al. 2007). Supersonic gas is also highly dissipative and without constant replenishment, damps within a crossing time. There are many sources of turbulent motions that can affect molecular clouds, such as galactic spiral shocks in which most giant molecular clouds form, supernovae, expanding HII regions, radiation pressure, cosmic ray streaming, Kelvin-Helmholtz and Rayleigh-Taylor instabilities, gravitational instabilities, and bipolar outflows from regions of star formation (Elmegreen \& Scalo 2004).

Supernova driven bubbles and turbulence in the galactic disk have been simulated by several groups. The work of de Avillez \& Breitschwerdt (2004) simulates the high resolution (down t0 1.5 pc scales) global structure of the ISM as a function of the supernova rate. Densities range over six orders of magnitude, $10^{-4} \leqslant n \leqslant 10^{2} \mathrm{~cm}^{-3}$ and multiphase density PDFs are given. Simulations of the global structure of a supernova lashed, multi-component, instellar medium (Tasker \& Bryan 2008; Wada \& Norman 2007) find that the density PDF follows a lognormal distribution. 
Supersonic turbulence produces hierarchical structure that can be described by a lognormal distribution (Vazquez-Semadeni 1994). A lognormal arises whenever the probability density of each new step in density increment in the turbulence is independent of the previous one. As an example, consider a medium that undergoes a series of random shocks whose strengths are uniformly distributed (Kevlahan \& Pudritz 2009). The density at any point is the product of the shock-induced, density jumps. Taking the log of this relation, and then the limit of a large number of shocks, the central limit theorem then shows that the log of the gas density should be normally distributed - hence the lognormal. It can also be shown mathematically that the convergence of the distribution for a finite number of shocks is very rapid - just 3 or 4 shocks will give a distribution that is very highly converged to a lognormal.

Lognormal behaviour for the CMF has been found in a wide variety of simulations using various types of codes (eg. SPH, AMR) and setups (driven or not driven, periodic boxes, initial uniform spheres, etc.). Early results showed that lognormal behaviour in periodic box simulations is independent of details on how the turbulence is driven (Klessen 2001).

It is interesting that lognormal distributions appear across science (eg. physics, biology, medicine, etc.), not just in fluid mechanics. The key difference between normal and lognormal distributions in general is that the former arises for additive processes, whereas the latter arise in multiplicative ones (Limpert et al. 2001). As a concrete example, consider a simple dice game where one first adds the values on the faces of two thrown dice - the distribution of results (ranging from 2 to 12) is a normal distribution whose mean is 7 . If one multiplies the two values however, the resulting distribution of numbers is highly skewed (ranging between 1 and 36) and is described by a lognormal.

Thus, lognormals characterize structure in the diffuse ISM as well as in denser molecular gas because shocks are the dominant process for configuring the gas. This is independent of exactly how the shocks are produced. Of physical significance are the mass of the peak of this distribution, and its width $\sigma_{o}$. The latter depends on both the thermal state of the gas as well as the rms Mach number of the turbulence. The standard deviation $\sigma_{o}$ is found from the simulations, and takes the form; $\sigma_{o}^{2}=\ln \left(1+b^{2} M^{2}\right)$ where $\mathrm{M}$ is the rms Mach number of the turbulence and $b \simeq 0.5$ is a fitting parameter (Padoan et al. 1997).

\subsection{Gravity and accretion}

Adding gravity to turbulence changes this distribution - a power-law tail appears at the high mass end of the simulated CMF (Li et al. 2003; Tilley \& Pudritz 2004). In the semi-analytic treatment of Padoan \& Nordlund (2002), the power-law arises because of the turbulence spectrum of the turbulence. In Hennebelle \& Chabrier (2008), a PressSchecter formalism is adopted to argue that a lognormal plus power-law behaviour is the consequence of imposing a star formation threshold (eg. Jeans' criterion) and gravity at high mass.

Much of the debate concerning the origin of the CMF and IMF has focused on whether stars form by competitive accretion (Bonnell et al. 2001) or by the gravitational collapse of discrete cores (Krumholz et al. 2005). Cluster formation simulations often use initial top-hat density profiles (uniform spheres) that are chosen to mimic the observed initial conditions of cluster forming clumps (eg. a hundred solar masses of material, at temperature of $10 \mathrm{~K}$, size of half a pc, and mean density of at least $10^{5} \mathrm{~cm}^{-3}$ ). Such simulated clumps start to undergo global gravitational collapse in less than a free-fall time (eg. Bonnell et al. (2001), Tilley \& Pudritz (2004)) as the turbulent energy is dissipated. The collapsing background ramps up the density of the gas including those in fluctuations such as the filaments. This drives up the accretion of gas into the filaments pushing some of them towards gravitational fragmentation. Sink-particles (taken as proxies for cores) 
first appear within the filaments. Cores will collapse more quickly than the collapsing background clump because they are denser and therefore have shorter free-fall times. The the collapse carries the collection of sink particles and filaments into the ever deepening potential well of the clump.

Cores are not isolated objects within filaments. Rather, they continue to undergo considerable filamentary accretion. The first objects to appear within most simulations generally become the most massive cores. In Banerjee et al. (2006), the rapid growth of the first, and most massive star by filamentary accretion was followed with an AMR (FLASH) code. These simulations showed that the filament was formed at the intersection of two sheets- shocks.

\subsection{Equations of state}

The thermal state of the bulk gas is a major factor in determining the local Jeans mass from point to point in molecular clouds. Both theory and simulations show that equation of state plays a very important role in controling the gravitational fragmentation of the gas. The local Jeans mass scales with the local temperature and density as $M_{J} \propto$ $T^{3 / 2} \rho^{1 / 2}$. For simple polytropic equations of state $P \propto \rho^{\gamma}$, the Jean's mass can be written purely as a power law of the density; $M_{J} \propto \rho^{3 / 2(\gamma-(4 / 3))}$. This scaling suggests that for $\gamma>4 / 3$, the Jeans mass increases with density, which puts an end to fragmentation. Indeed, simulations show that strong fragmentation prevails for $\gamma \leqslant 1$, gets progressively weaker for $\gamma>1$, and stops altogether for $\gamma>1.4$ ( $\mathrm{Li}$ et al. 2003).

Local energy sources can raise the fragmentation mass by changing the temperature of the region. Thus, the accretion luminosity released by massive stars in particular, must reduce the degree of fragmentation within a localized region around such a heat source, as has been demonstrated by several groups (Krumholz et al. 2007). For massive stars, this region is limited in extent - roughly $1000 \mathrm{AU}$ or so (see $\S 4$ ).

\subsection{Magnetic fields}

Magnetic fields play several different roles in star formation. As has long been known, they can control the gravitational stability of a gas if their energy density exceeds that of gravity. This is formalized by mass to flux ratio; $\Gamma=2 \pi \sqrt{G} \Sigma / B=1.4 \beta^{1 / 2} n_{J}^{1 / 3}$ where $n_{J}$ is the number of Jeans masses and $\beta$ is the ratio of gas to magnetic pressure. The fragmentation of a uniform cloud is highly suppressed for subcritical clouds $(\Gamma<1)$. Simulations of slightly supercritical clumps in uniform magnetic fields show that the field channels the collapse into large sheets that are perpendicular to the direction of the field. Slightly more supercritical clouds however, break up into more substructure including filaments (Tilley \& Pudritz 2007). Turbulence creates a very broad range of magnetizations of cores. This is because shocks sweep material along field lines where it accumulates in filaments - increasing the mass to flux ratio in those regions, and greatly reducing the mass to flux ratio in the more diffuse zones left behind. Simulations show that initially supercritical magnetized clouds results in cores that range from critical to strongly supercritical (Padoan \& Nordlund 2002; Tilley \& Pudritz 2007), in agreement with the observations (Crutcher 2007). This may also account for the fact that magnetic fields are more dominant in the diffuse gas than in molecular gas.

When turbulence is added to a subcricital cloud, the column density PDF of a cloud is a lognormal but with a very small standard deviation. When ambipolar diffusion is added, the lognormal broadens considerably (Nakamura \& Li 2008)). Supercritical subregions can form within the subcritical cloud, and it is within these regions that star formation can proceed, albeit at a very heavily reduced rate. 
The second major aspect of magnetic fields is that twisted fields exert torques on gas and can therefore transport angular momentum away from spinning bodies. The origin of angular momentum in supersonic turbulence is that oblique shocks produce spinning cores (Jappsen \& Klessen 2004; Tilley \& Pudritz 2007)). Turbulence simulations produce a broad distribution of angular momenta of cores ranging over nearly two orders of magnitude. This implies that a broad distribution of disk sizes should result from the collapse of these systems - from very small to very large disks. The distribution of angular momentum vectors is also quite varied and is not particularly aligned with the filament principal axes.

Magnetic fields participate in several kinds of braking. On the level of the cores, torsional Alfvén waves have long been known to be able to extract significant amounts of angular momentum Basu \& Mouschovias (1994). The collapse of rotating cores produces magnetized outflows as demonstrated in a variety of initial core models; cylinders (Tomisaka 2002), Bonner-Ebert spheres (Banerjee \& Pudritz 2006), uniform spheres (Hennebelle \& Fromang 2008), and singular isothermal spheres (Mellon \& Li 2008). Early outflows could sweep up significant amounts of material and have been implicated as the basic physics in the CMF to IMF efficiency factor $\epsilon$ (see $\S 4.3$ ).

Finally, the combination of radiative and MHD effects (RMHD) limits the fragmentation of cluster-forming gas. Attempts to model RMHD on cluster scales have been made by Price \& Bate (2009) who used Euler potentials to approximate the MHD in SPH. This work shows that the MHD in their code strongly supresses fragmentation even for clouds that are fairly supercritical (eg. $\Gamma=3$ ). On smaller scales, grid-based RMHD methods applied to collapse and outflows show that radiative heating (in flux limited diffusion limit) makes substantial changes to the extent of outflows (Commerçon et al. 2010; Tomida et al. 2010).

\section{Feedback: from the CMF to the IMF}

\subsection{Stellar dynamics and filamentary accretion}

The collapse of $10^{2} M_{\odot}$ clumps pulls both the material in the filaments and the sink particles deeper into the central potential well. The sink-sink interactions, which are modeled to be N-body gravity then come into play and are responsible for the creation of a small stellar cluster. The competitive accretion scenario predicts that these objects compete for gas in the dense centre of the gravitational potential well, resulting in an IMF (Bonnell et al. 2001). For larger clumps $\left(10^{3}-10^{4} M_{\odot}\right)$ the evolution of a cluster forming region is a hierarchical process in which subclusters form and ultimately merge (Bonnell et al. 2003). The resulting stellar interactions are frequent and close enough to truncate protostellar disks.

Sink particles as implemented by Federrath et al. (2010) have the additional desirable feature that they form in local potential minima giving them a more hydrodynamic character. This has an important consequence. As opposed to competitive accretion onto particles in a general potential well, filamentary accretion largely ends when the N-body interactions become strong enough to kick the sink particles out of their feeding filaments Evidence for filamentary accretion is seen in the simulations of Duffin et al. (2010a), and shown in Figure 1 (featuring the collapse of a $100 M_{\odot} \simeq 100 M_{J}$ initial tophat clump). The first frame, shows that sink particles form in filaments. The second shows that dynamical interactions have started between them as the collapse of the clump proceeds, and the third shows the dynamical end state. The final frame shows that the accretion histories of each particle shuts off when when dynamical interactions become important. 


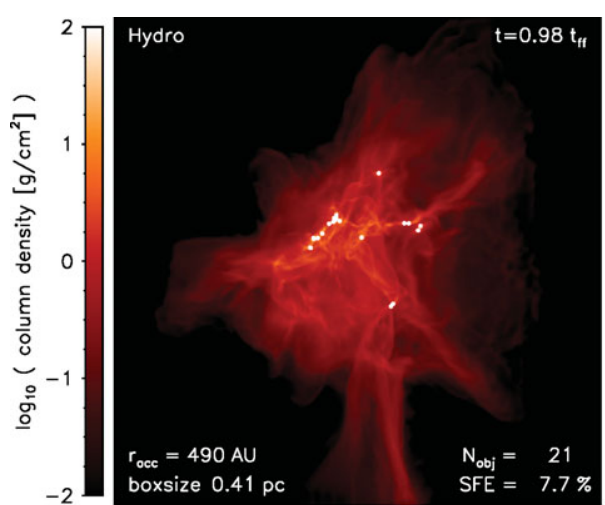

Figure 1a

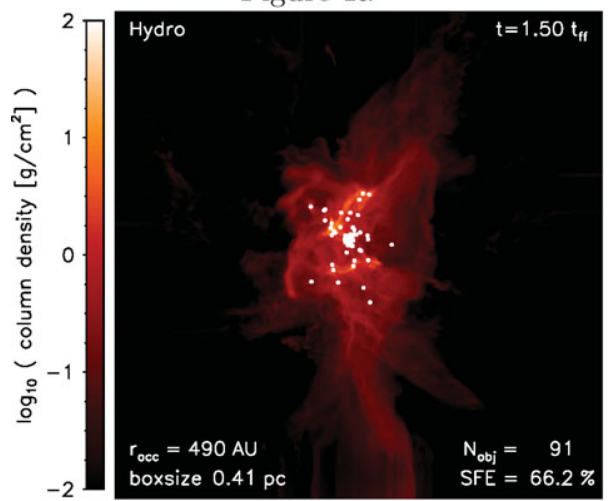

Figure 1c

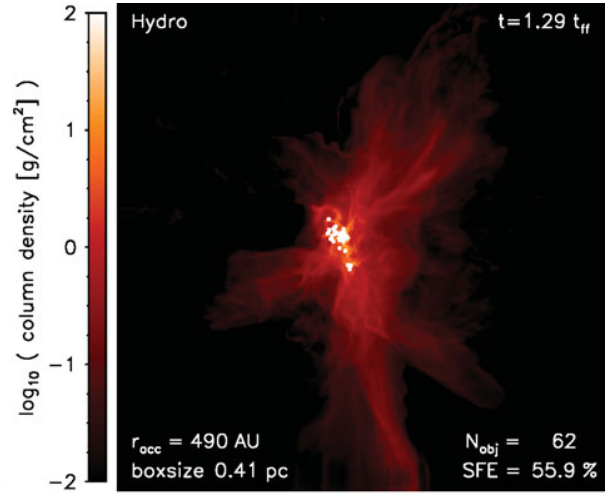

Figure $1 b$

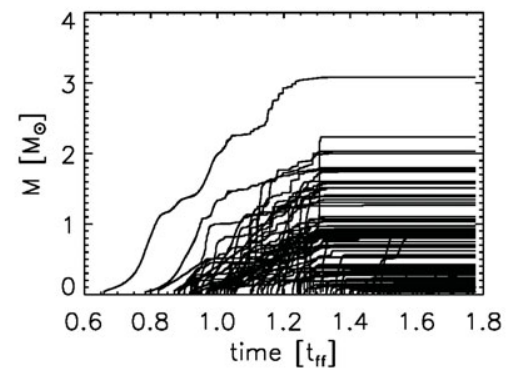

Figure 1d

Figure 1. Cluster formation and filamentary accretion. Top left: sink particles form in filaments. Top right: sink particles begin dynamical interaction. Bottom left: cluster dynamics, accretion has ceased. Bottom right: mass accretion history for each sink particle - accretion ends when dynamical interactions begin (Duffin et al. 2010a).

\subsection{Radiative feedback}

Comprehensive 2D simulations of massive star formation including full frequency, radiative feedback effects and dust are presented by Yorke \& Sonnhalter (2002). For cluster formation, a 3D treatment of radiative transfer in a highly inhomogenous medium is essential. Since Krumholz et al. (2007), it has become widely appreciated that radiative heating of the gas is needed to prevent excessive gravitational fragmentation. Radiative heating of the gas prevents the filaments from fragmenting as much as they might. Their gas drains primarily into the central massive forming disk and star, and fragmentation out to 1000 AU scales is prevented. These calculations still invoke grey, flux limited diffusion so there is still a need to examine the role of different frequency regimes in this process. Simulations by Bate (2009) confirm this picture using SPH techniques - wherein suppression of brown dwarfs by a factor of 4 is often observed compared to simulations without radiative feedback. The ionizing radiation from massive young stars becomes important during cluster formation as HII regions drive hot ioinized flows in the cluster environment. Results show that companions to massive stars limit the growth of the latter by a starvation effect (Peters et al. 2010). 


\subsection{Outflows}

Outflows carry substantial amounts of mechanical energy which, if coupled into clump dynamics, could affect the IMF. Two aspects of feedback are important outflows in a cluster region (Klessen et al. 2009). The first is at the core level, in which an outflow removes the collapsing gas at some efficiency. If this efficiency is quite high (so that $\epsilon \simeq 1 / 3$ ), then one may resolve the hypothesized conversion of the CMF to IMF (Matzner $\&$ McKee 2000). Simulations show that the efficiency may be much lower (Duffin et al. $2010 \mathrm{~b})$. The second is at the level of the clump and the question as to whether or not the collection of outflows can continue to excite turbulence and thereby regulate cluster formation Norman \& Silk (1980).

The situation at the local level is still unclear. Simulations by Banerjee et al. (2007) show that supersonic turbulence is not driven by magnetized jets. At the level of clumps, Nakamura \& Li (2008) included ambipolar diffusion and outflows in a cloud that is nearly critical ( $\Gamma=1.1$ initially), and found, not unexpectedly, that the magnetic fields regulated a rather slow rate of star formation. On the other hand, Wang et al. (2010) used a mass to flux of $\Gamma=1.4$ in a code without $\mathrm{AD}$, and observed long time regulation of the cluster formation by outflows which maintained turbulence. These results may all depend upon the limited modeling of the the full dynamics of outflows that have been incorporated into the simulations.

\section{Synthesis: the IMF of clusters}

Numerical simulations have become the primary tool with which to investigate the origin of the IMF in clusters. Turbulence, the equation of state, and gravity play the key roles of filamenting the gas and breaking it into accreting cores describable by a lognormal distribution with high mass power-law tail. Radiative feedback controls fragmentation rates at the low mass end of the IMF whereas feedback by outflows may not be as efficient as previously claimed in converting the CMF to the IMF. Filamentary structure from clouds to clumps may turn out to play a key role in the entire process.

\section{References}

Alves, J., Lombardi, M., \& Lada, C. J. 2007, A\& A, 462, L17

André, P., et al. 2010, A\& A, 518, L102+

Banerjee, R., Klessen, R. S., \& Fendt, C. 2007, ApJ, 668, 1028

Banerjee, R. \& Pudritz, R. E. 2006, ApJ, 641, 949

Banerjee, R., Pudritz, R. E., \& Anderson, D. W. 2006, MNRAS, 373, 1091

Basu, S. \& Mouschovias, T. C. 1994, ApJ, 432, 720

Bate, M. R. 2009, MNRAS, 392, 1363

Bonnell, I. A., Bate, M. R., Clarke, C. J., \& Pringle, J. E. 2001, MNRAS, 323, 785

Bonnell, I. A., Bate, M. R., \& Vine, S. G. 2003, MNRAS, 343, 413

Bonnell, I. A., Larson, R. B., \& Zinnecker, H. 2007, in Protostars and Planets V, ed. B. Reipurth, D. Jewitt, \& K. Keil, 149-164

Cambrésy, L. 1999, A\&BA, 345, 965

Chabrier, G. 2003, PASP, 115, 763

Commerçon, B., Hennebelle, P., Audit, E., Chabrier, G., \& Teyssier, R. 2010, A\&SA, 510, L3+ Crutcher, R. 2007, in Star Formation, Then and Now

de Avillez, M. A. \& Breitschwerdt, D. 2004, A\&A, 425, 899

Duffin, D. F., Federrath, C., Pudritz, R. E., Banerjee, R., \& Klessen, R. 2010a, in preparation

Duffin, D. F., Pudritz, R. E., Banerjee, R., \& Seifried, D. 2010b, in preparation

Elmegreen, B. \& Scalo, J. 2004, Ann. Rev. Astron. Astrophys., 42, 211 
Federrath, C., Banerjee, R., Clark, P. C., \& Klessen, R. S. 2010, ApJ, 713, 269

Fiege, J. D. \& Pudritz, R. E. 2000, MNRAS, 311, 105

Hennebelle, P. \& Chabrier, G. 2008, ApJ, 684, 395

Hennebelle, P. \& Fromang, S. 2008, A\& $A$, 477, 9

Henning, T., Linz, H., Krause, O., Ragan, S., Beuther, H., Launhardt, R., Nielbock, M., \& Vasyunina, T. 2010, $A \& A, 518$, L95+

Jappsen, A. \& Klessen, R. S. 2004, $A \& A A, 423,1$

Johnstone, D. \& Bally, J. 2006, ApJ, 653, 383

Kainulainen, J., Beuther, H., Henning, T., \& Plume, R. 2009, A\&SA, 508, L35

Kevlahan, N. \& Pudritz, R. E. 2009, ApJ, 702, 39

Klessen, R. S. 2001, ApJ, 556, 837

Klessen, R. S. \& Burkert, A. 2001, ApJ, 549, 386

Klessen, R. S., Krumholz, M. R., \& Heitsch, F. 2009, ArXiv e-prints

Kroupa, P. 2002, Science, 295, 82

Krumholz, M. R., Klein, R. I., \& McKee, C. F. 2007, ApJ, 656, 959

Krumholz, M. R., McKee, C. F., \& Klein, R. I. 2005, Nat, 438, 332

Larson, R. B. 2007, Reports on Progress in Physics, 70, 337

Li, Y., Klessen, R. S., \& Mac Low, M. 2003, ApJ, 592, 975

Limpert, E., Stahel, W. A., \& Abbt, M. 2001, Bioscience, 51, 341

Mac Low, M.-M. \& Klessen, R. S. 2004, Reviews of Modern Physics, 76, 125

Matzner, C. D. \& McKee, C. F. 2000, ApJ, 545, 364

McKee, C. F. \& Ostriker, E. C. 2007, ARA $\mathscr{G} A, 45,565$

Mellon, R. R. \& Li, Z. 2008, ApJ, 681, 1356

Meyer, M. R., Adams, F. C., Hillenbrand, L. A., Carpenter, J. M., \& Larson, R. B. 2000, Protostars and Planets IV, 121

Miller, G. E. \& Scalo, J. M. 1979, ApJS, 41, 513

Motte, F., Andre, P., \& Neri, R. 1998, A\& A, 336, 150

Nagasawa, M. 1987, Progress of Theoretical Physics, 77, 635

Nakamura, F. \& Li, Z. 2008, ApJ, 687, 354

Norman, C. \& Silk, J. 1980, ApJ, 238, 158

Ostriker, E. C., Gammie, C. F., \& Stone, J. M. 1999, ApJ, 513, 259

Padoan, P., Juvela, M., Goodman, A. A., \& Nordlund, A. 2001, ApJ, 553, 227

Padoan, P. \& Nordlund, A. 2002, ApJ, 576, 870

Padoan, P., Nordlund, A., \& Jones, B. J. T. 1997, MNRAS, 288, 145

Paresce, F. \& De Marchi, G. 2000, ApJ, 534, 870

Peters, T., Banerjee, R., Klessen, R. S., Mac Low, M., Galván-Madrid, R., \& Keto, E. R. 2010, ApJ, 711, 1017

Porter, D., Pouquet, A., \& Woodward, P. 1994, Phys. Fluids, 6, 2133

Price, D. J. \& Bate, M. R. 2009, MNRAS, 398, 33

Salpeter, E. E. 1953, Annual Review of Nuclear and Particle Science, 2, 41

Tasker, E. J. \& Bryan, G. L. 2008, ApJ, 673, 810

Taylor, A. R., et al. 2003, AJ, 125, 3145

Tilley, D. A. \& Pudritz, R. E. 2004, MNRAS, 353, 769

-. 2007, MNRAS, 382, 73

Tomida, K., Tomisaka, K., Matsumoto, T., Ohsuga, K., Machida, M. N., \& Saigo, K. 2010, ApJL, 714, L58

Tomisaka, K. 2002, ApJ, 575, 306

Vazquez-Semadeni, E. 1994, ApJ, 423, 681

Vazquez-Semadeni, E., Passot, T., \& Pouquet, A. 1995, ApJ, 441, 702

Wada, K. \& Norman, C. A. 2007, ApJ, 660, 276

Wang, P., Li, Z., Abel, T., \& Nakamura, F. 2010, ApJ, 709, 27

Yorke, H. W. \& Sonnhalter, C. 2002, ApJ, 569, 846

Zinnecker, H., McCaughrean, M. J., \& Wilking, B. A. 1993, in Protostars and Planets III, ed. E. H. Levy \& J. I. Lunine, 429-495 